\title{
Reliability-aware iterative detection scheme (RAID) for distributed IDM space-time codes in relay systems
}

\author{
Florian Lenkeit ${ }^{*}$, Dirk Wübben and Armin Dekorsy
}

\begin{abstract}
In this article, distributed interleave-division multiplexing space-time codes (dIDM-STCs) are applied for multi-user two-hop decode-and-forward (DF) relay networks. In case of decoding errors at the relays which propagate to the destination, severe performance degradations can occur as the original detection scheme for common IDM-STCs does not take any reliability information about the first hop into account. Here, a novel reliability-aware iterative detection scheme (RAID) for dIDM-STCs is proposed. This new detection scheme takes the decoding reliability of the relays for each user into account for the detection at the destination. Performance evaluations show that the proposed RAID scheme clearly outperforms the original detection scheme and that in certain scenarios even a better performance than for adaptive relaying schemes can be achieved.
\end{abstract}

\section{Introduction}

During the last decade, cooperative communications and especially relaying has been a promising and constantly growing field of research. While, in the first place, the main purpose for introducing relays into wireless communication systems was the reduction of path losses between communicating nodes, relay systems also offer spatial diversity and, therefore, allow for the adoption of diversity exploiting techniques known from multi-antenna (Multiple Input - Multiple Output, MIMO) systems. MIMO systems have been shown to allow for a significantly higher performance in terms of throughput or robustness against fading than their single-antenna counterparts, which is mainly due to the exploitation of space for further degrees of freedom in addition to time and frequency $[1,2]$.

In this study, we consider a two-hop relaying system in which multiple sources transmit to one destination via a number of parallel relays. In such a system, the distributed relays can be grouped into a so-called Virtual Antenna Array (VAA), which can then be used to jointly process data, exploiting receive or transmit diversity [3]. Due to the distributed fashion of the VAA it can not be assumed that all nodes within the VAA can exchange arbitrarily

\footnotetext{
*Correspondence: lenkeit@ant.uni-bremen.de

Department of Communications Engineering, University of Bremen, Bremen, Germany
}

much information as this would severely increase the signaling overhead and subsequently decrease the overall throughput. Hence, it is not possible to achieve perfect receive cooperation among all nodes within the VAA with justifiable effort. However, since the focus of this study is on the exploitation of transmit diversity, no receive cooperation at all is assumed and, thus, signaling among the relays is avoided. This means that each relay might estimate the source information differently. Furthermore, besides the limited exchange of information, it is also a challenging task to keep all nodes perfectly synchronous in terms of timing and frequency. Asynchronism among the nodes of the VAA or, in general, among different VAAs can lead to severe performance degradations for transmission schemes which require strict orthogonality among the transmitting nodes.

One of the most prominent transmit cooperation techniques applied to VAAs is Space-Time Coding. SpaceTime Coding evolved in the context of MIMO systems and is a very efficient transmit diversity exploiting strategy if no Channel State Information (CSI) is available at the transmitter $[4,5]$. The idea behind Space-Time Coding is to exploit space and time as two dimensions for coding. The first applications of Space-Time Codes (STCs) to relaying systems was done in [6] and later in [7]. Since these approaches were direct adoptions of

\section{是 Springer}

C 2013 Lenkeit et al: licensee Springer. This is an Open Access article distributed under the terms of the Creative Commons Attribution License (http://creativecommons.org/licenses/by/2.0), which permits unrestricted use, distribution, and reproduction in any medium, provided the original work is properly cited. 
Alamouti's scheme and its corresponding generalization, they principally require perfect orthogonality among the relay signals. In $[8,9]$ a STC approach based on the nonorthogonal multiple access scheme Interleave-Division Multiple Access (IDMA) [10] has been presented. This Interleave-Division Multiplexing Space-Time Code (IDMSTC) does not require any synchronization among the transmit antennas which makes it a promising technique for relay systems. Consequently, the IDM-STC has been applied to relay systems in a distributed fashion using uncoded transmission and the Decode-and-Forward (DF) relaying protocol [11]. In [12], the principles of distributed IDM-STCs (dIDM-STCs) have been extended to coded systems and further relay protocols like Amplify-andForward (AF) and Decode-Estimate-and-Forward (DEF). It has been shown, that the dIDM-STC is in fact a good choice for relaying systems due to its flexibility regarding code rate and number of transmitting nodes and due to its robustness against asynchronisms. However, it was also pointed out that non-perfect decoding at the relays has to be taken into account at the destination as it leads to error propagation, which is a general problem of DF since it can severely degrade the overall performance.

One possibility to deal with erroneous decoding at the relays is to adaptively select only those relays to forward to the destination which have decoded correctly [7]. However, since erroneous relays may still contribute to the transmission, it does not seem reasonable to discard erroneously decoded messages. Instead, all relays should forward to the destination and the detector should handle the correct as well as the erroneous messages properly. In [13] a detector for uncoded DF was presented which applies a weighting of the relay messages according to the reliability of the whole source-relay-destination link. For that, all S-R-D links were modeled by equivalent Gaussian channels whose SNRs are dependent on the bit error probabilities on both hops. The messages over different S-R-D links were then weighted according to the respective equivalent SNR and finally combined. In $[14,15]$ distributed turbo codes in DF systems were investigated. In these studies, the first hop transmission was modeled using a Binary Symmetric Channel (BSC) with a crossover probability dependent on the first hop transmission quality, i.e., the bit error probability at the relay. The BSC model was then used in order to weight the exchanged information between the two constituent decoders at the destination. In [16] we presented a modified detection strategy for dIDM-STC, adopting the ideas from $[13,14]$ and incorporating the decoding reliability of the relays into the detection at the destination. This was achieved by estimating the decoding reliabilities at the relays and signaling this information to the destination. The reliability information of all relays was then used for a weighted combining of the relay messages. As a result, a significant performance improvement was achieved compared to the common detection scheme for dIDM-STC from [12]. However, it could also be seen that the actual usage of the available information at the destination was not yet optimal as the results indicated a performance gap compared to adaptive relaying schemes in which only correct relays forward to the destination.

In this article, we present a novel Reliability-Aware Iterative Detection scheme (RAID) for distributed IDM-STCs in Decode-and-Forward relay systems. The main idea of this scheme is to distinguish between the detection of the relay messages and the subsequent estimation of the source messages. First, a grouping of the relays is introduced and the relays are separated by their decoding success. While the signals from the successful relays are combined and jointly decoded, as for the common detector, the signals from the erroneous relays are all processed separately. The goal here is to firstly estimate the relay messages as good as possible. This approach is different to [16] where all relay signals were combined during the iterative detection, directly estimating the source information. After the final iteration of the iterative detection process, the estimates of the messages of the correct relays and the estimates of the messages of the erroneous relays are combined using a weighting operation similar to [16] in order to estimate the source messages. In contrast to [16], here the weighting is carried out after the iterative detection process, i.e., after firstly estimating the relay messages. While first ideas of this new detection scheme have been presented in [17], here the RAID scheme is extended to the multi-user case. We present more detailed derivations as in [17] and also give much further insight especially into the determination of the error probabilities at the relays. We will show that estimating the error probabilities in a practical system leads to almost no performance loss compared to a perfect knowledge. Moreover, extensive comparisons with other detection schemes like the common detection scheme or the adaptive scheme are given and discussed for singleuser as well as for multi-user systems. As will be shown, the proposed RAID scheme achieves significant performance improvements compared to the common detection and to the strategy in [16]. It even performs better than the adaptive relaying scheme in certain scenarios, as it also exploits information from erroneous relays which is not the case for the adaptive scheme.

The remainder of this article is structured as follows. In Section 2 the system model is presented and its components are discussed in detail. Section 3.1 discusses the common detection scheme and addresses the main problems which arise in the context of relay systems. Section 4 is dedicated to the new proposed RAID scheme. First, an equivalent transmission model for the source relay transmission is presented and discussed, which is the basis 
for the formulation of the new detector. Then, the new RAID scheme is presented in detail. In Section 5 numerical results are given and the RAID scheme is compared to the conventional IDM-STC detection scheme as well as to an adaptive scheme. In Section 6, finally, some conclusions are drawn.

The nomenclature for this article is as follows. Scalars are denoted by small letters $a$, while column vectors are denoted by bold letters $\mathbf{a}=\left[a_{1} \ldots a_{N}\right]^{\mathrm{T}}$ and matrices by bold capital letters $\mathbf{A}=\left[a_{1,1} \ldots a_{1, N} ; \ldots ; a_{M, 1} \ldots a_{M, N}\right]$. Interleaved sequences are denoted by a dash a' and double interleaved sequences by two dashes a". Log-likelihood-ratios (LLRs) of scalar $a$ and of elements of a vector a are denoted by $\boldsymbol{\Lambda}_{a}=\log \left(\frac{\operatorname{Pr}\{a=0\}}{\operatorname{Pr}\{a=1\}}\right)$ and $\boldsymbol{\Lambda}_{a}=\left[\boldsymbol{\Lambda}_{a_{1}}, \ldots, \boldsymbol{\Lambda}_{a_{N}}\right]$, respectively. The expectation of a random process $X$ is expressed as $\mathrm{E}\{X\}$ and its variance as $\sigma_{\mathrm{X}}^{2}$. The $\mathrm{L}_{2}$-norm of a vector a is denoted by $\|\mathbf{a}\|$ and $\mathbb{F}_{2}$ is the gallois field $\mathrm{GF}(2)$.

\section{System model}

\subsection{Overview}

A two-hop relay system as depicted in Figure 1 is considered. Multiple sources $S_{m}, 1 \leq m \leq M$, communicate with one common destination $D$ via $N$ parallel relays $R_{n}, \quad 1 \leq n \leq N$ performing Decode-and-Forward. No direct links from sources to destination are assumed and the discrete-time channel impulse responses from $S_{m}$ to $R_{n}$ and from $R_{n}$ to $D$ are given by $\mathbf{h}_{m, n}$ and $\mathbf{g}_{n}$, respectively. Frequency-selective block Rayleigh fading channels $\tilde{\mathbf{h}}_{m, n}$ and $\tilde{\mathbf{g}}_{n}$ with $L_{\mathrm{h}}$ and $L_{\mathrm{g}}$ i.i.d channel taps are assumed and the path loss on each hop is given by $d^{\epsilon}$ such that

$$
\begin{aligned}
\mathbf{h}_{m, n} & =d^{-\frac{\epsilon}{2}} \tilde{\mathbf{h}}_{m, n} \\
\mathbf{g}_{n} & =d^{-\frac{\epsilon}{2}} \tilde{\mathbf{g}}_{n},
\end{aligned}
$$

where $d$ denotes the distance between the corresponding nodes and $\epsilon$ is the path loss exponent. The channel

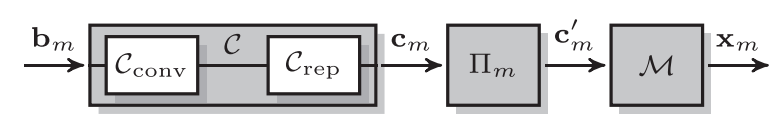

Figure 2 Structure of source $S_{m}$ : common IDMA transmitter structure consisting of channel encoder $\mathcal{C}$, interleaver $\Pi_{m}$ and symbol mapper $\mathcal{M}$.

impulse responses are normalized such that the total received power depends only on the path loss but not on $L_{\mathrm{h}}$ and $L_{\mathrm{g}}$, respectively, i.e., $\mathrm{E}\left\{\left\|\tilde{\mathbf{h}}_{m, n}\right\|^{2}\right\}=\mathrm{E}\left\{\left\|\tilde{\mathbf{g}}_{n}\right\|^{2}\right\}=$ 1. Also, each receiver, i.e., $R_{n}$ and $D$ experiences additive white gaussian noise (AWGN) of power $\sigma_{n}^{2}$. Due to the half-duplex constraint, the transmission time can be divided into a source-relay phase in which the sources broadcast their information to the relays and a relaydestination phase in which the relays simultaneously forward the processed information of all sources to the destination. Both phases are described in detail in the subsequent paragraphs.

\subsection{Source-relay phase}

In the first phase, the sources simultaneously broadcast their information to the relays applying IDMA [10]. Figure 2 shows the transmitter structure of the source $S_{m}$, where the binary information sequence $\mathbf{b}_{m} \in \mathbb{F}_{2}^{L_{\mathrm{b}}}$ of length $L_{\mathrm{b}}$ is encoded by a code $\mathcal{C}$ of rate $R_{\mathrm{c}}=R_{\mathrm{c}, \text { conv }} \cdot R_{\mathrm{c} \text {,rep }}$ consisting of a serial concatenation of a convolutional code $\mathcal{C}_{\text {conv }}$ of rate $R_{\text {c,conv }}$ and a repetition code $\mathcal{C}_{\text {rep }}$ of rate $R_{\mathrm{c}, \text { rep }}$. The resulting coded sequence $\mathbf{c}_{m} \in \mathbb{F}_{2}^{L_{\mathrm{c}}}$ of length $L_{\mathrm{c}}$ is interleaved by a user specific interleaver $\Pi_{m}$ resulting in the interleaved code sequence $\mathbf{c}_{m}^{\prime} \in \mathbb{F}_{2}^{L_{\mathrm{c}}}$. Finally, the interleaved code bits are mapped onto symbols from the normalized alphabet $\mathcal{A}$ resulting in the transmit sequence $\mathbf{x}_{m} \in \mathcal{A}^{L_{\mathrm{x}}}$ of length $L_{\mathrm{x}}$ with $\sigma_{\mathrm{x}}^{2}=1$. The symbols $\mathbf{x}_{m}$ are then broadcasted to all relays.

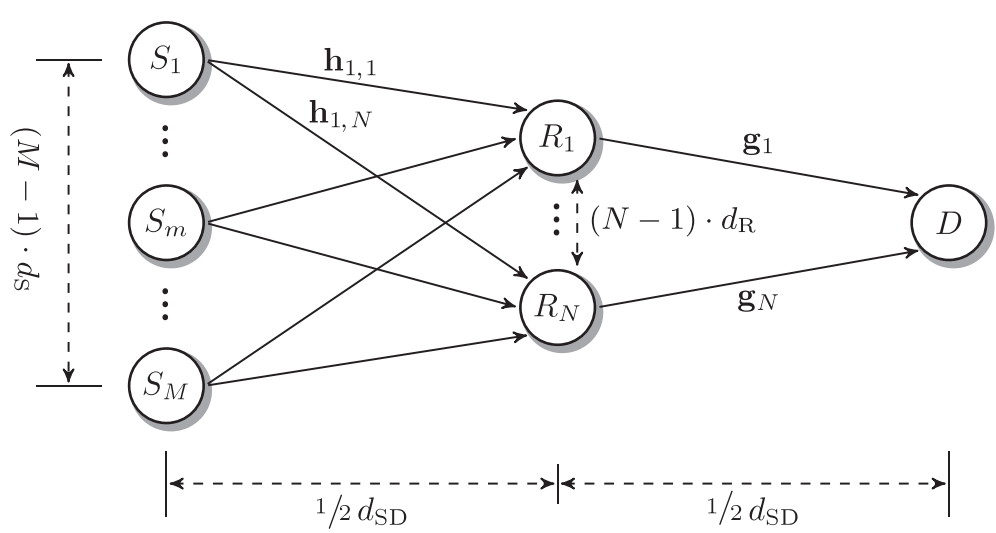

Figure 1 Topology of the considered multi-user two-hop relay system. Sources $S_{m}$ and relays $R_{n}$ are distributed equidistantly with spacings $d_{s}$ and $d_{R}$, respectively. Relays are located directly in the middle between sources and destination. 
The received signal $\mathbf{y}_{n}$ at relay $R_{n}$ is given by the superposition of all source signals $\mathbf{x}_{m}$ convolved with the corresponding channel impulse responses $\mathbf{h}_{m, n}$ plus additive white gaussian noise $\mathbf{n}_{n} \in \mathbb{C}^{L_{\mathrm{x}}+L_{\mathrm{h}}-1}$ of power $\sigma_{\mathrm{n}}^{2}$ as

$$
\mathbf{y}_{n}=\sum_{m=1}^{M} \mathbf{H}_{m, n} \mathbf{x}_{m}+\mathbf{n}_{n}
$$

where $\mathbf{H}_{m, n} \in \mathbb{C}^{\left(L_{\mathrm{x}}+L_{\mathrm{h}}-1\right) \times L_{\mathrm{x}}}$ is the convolutional matrix of $\mathbf{h}_{m, n}$. In Figure 3, the structure of relay $R_{n}$ is shown. First, in order to separate the user signals $\mathbf{x}_{m}$, IDMA multi-user detection (MUD) is performed using the iterative soft-RAKE algorithm [10]. Since for soft-RAKE detection all multipath propagations of each signal $\mathbf{x}_{m}$ are resolved separately, in total $M \cdot L_{\mathrm{h}}$ different layers have to be resolved at each relay. As a useful measure for later investigations, the load on the first hop $\beta_{\mathrm{SR}}$ is introduced. To account for the separate detection of all multipath propagations by the soft-RAKE detection, it is defined here in terms of layers as

$$
\beta_{\mathrm{SR}}=M \cdot L_{\mathrm{h}} \cdot R_{\mathrm{c}}
$$

By this definition, the load is not only dependent on the number of users $M$ and the overall code rate $R_{\mathrm{c}}$, but also on the number of channel taps $L_{\mathrm{h}}$, i.e., the degree of frequency selectivity of the channel. An alternative to softRAKE detection is multi-layer APP detection [18], which avoids gaussian approximation of multi-user interference and, hence, allows for higher bandwidth efficiencies. But since the complexity of multi-layer APP detection grows exponentially with the number of users $M$ and the number of channel taps $L_{\mathrm{h}}$, i.e., $\mathcal{O}\left(2^{M L_{\mathrm{h}}}\right)$, it is not suited for the investigated system.

The MUD at relay $R_{n}$ delivers LLRs $\Lambda_{\mathrm{b}_{m}}^{R_{n}}$ for the user information words $\mathbf{b}_{m}$. After hard decision, the estimates $\hat{\mathbf{b}}_{m}^{R_{n}}=\mathcal{Q}\left(\boldsymbol{\Lambda}_{\mathrm{b}_{m}}^{R_{n}}\right)$ form the relay information words $\mathbf{b}_{m, n}$ as

$$
\hat{\mathbf{b}}_{m}^{R_{n}} \rightarrow \mathbf{b}_{m, n}
$$

where $\mathbf{b}_{m, n}$ denotes the relay message at relay $R_{n}$ with respect to source $S_{m}$. Note that due to decoding errors at the relay, the information words $\mathbf{b}_{m}$ and $\mathbf{b}_{m, n}$ might be different from each other. To cope with this problem, the following strategies are possible:

- Decoding errors at the relays are not detected and the decoding at the relays is assumed to be perfect. Subsequently, all $M$ relay messages $\mathbf{b}_{m, n}$ of relay $R_{n}$ are forwarded to the destination. This is done for the common dIDM-STC detection.

- Decoding errors are detected by exploiting a cyclic redundancy check $(\mathrm{CRC})$ code and only correct information words, i.e., $\mathbf{b}_{m, n}=\mathbf{b}_{m}$, are forwarded to the destination. This is done for adaptive relaying which is discussed in more detail in Section 3.2.

- The decoding error probability at the relays is estimated, e.g., as described in Section 4.1, and the transmit power for the relay messages is adjusted according to the decoding error probability at the corresponding relay. That means, the higher the error probability at the relay, the lower the corresponding transmit power. This is done for adaptive relaying with power allocation. This scheme is not considered further in this study, as it basically has the same problems as the common dIDM-STC detection scheme, which are described in Section 3.1.

- Decoding errors are detected as for the adaptive relaying scheme. However, in contrast to the adaptive scheme, erroneous decoding at the relays is handled by the detector at the destination and not by the relays. Hence, all $M$ information words $\mathbf{b}_{m, n}$ are transmitted with the same power to the destination, regardless of the decoding success. Additionally, the outcomes of the CRC checks w.r.t. the sources $S_{m}$ are signaled to the destination where they are used later on by the new proposed detection scheme.

For non-adaptive relaying, all relay information words $\mathbf{b}_{m, n}$ are encoded using the same channel code $\mathcal{C}$ as the sources and interleaved by the user specific interleavers $\Pi_{m}$. In addition to the user specific interleaving, the sequences $\mathbf{c}_{m, n}^{\prime}$ are additionally interleaved using a relay specific interleaver $\Pi_{\mathrm{r}, n}$, such that a unique interleaver tuple $\left(\Pi_{m} ; \Pi_{\mathrm{r}, n}\right)$ is assigned to each of the $M$. $N$ interleaved sequences $\mathbf{c}_{m, n}^{\prime \prime}$ across all $N$ relays. These

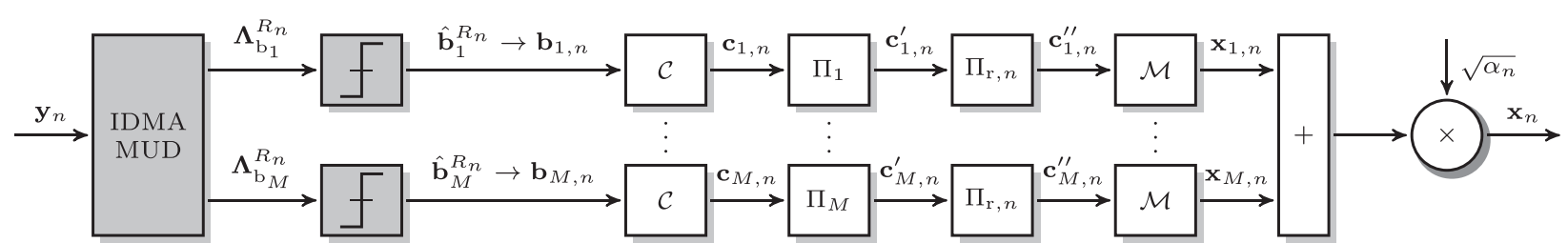

Figure 3 Structure of relay $R_{n}$ consisting of IDMA multi-user detection (MUD) and $M$ parallel IDMA encoding branches each containing a user specific and a relay specific interleaver in series. 
interleaved sequences are mapped onto symbols from the same alphabet $\mathcal{A}$ and summed up. Finally, a scaling by $\sqrt{\alpha_{n}}=\sqrt{\frac{1}{M}}$ ensures $\sigma_{\mathrm{x}_{n}}^{2}=1$ independent of the number of supported users $M$, resulting in the relay transmit signal

$$
\mathbf{x}_{n}=\sqrt{\alpha_{n}} \sum_{m=1}^{M} \mathbf{x}_{m, n} .
$$

\subsection{Relay-destination phase}

In the second phase, the transmit signals $\mathbf{x}_{n} \in \mathcal{A}^{L_{\mathrm{x}}}$ of all relays are broadcasted simultaneously to the destination $D$. Under the assumption of perfect decoding at all relays, each user message $\mathbf{b}_{m}$ is transmitted from all $N$ relays and a distributed IDM-STC is formed across the $N$ relays. The receive signal $\mathbf{y}$ at the destination consists of the superposition of the relay signals $\mathbf{x}_{n}$ convolved with the corresponding channel impulse responses $\mathbf{g}_{n}$ plus additive white gaussian noise $\mathbf{n} \in \mathbb{C}^{L_{\mathrm{x}}+L_{\mathrm{g}}-1}$ as

$$
\mathbf{y}=\sum_{n=1}^{N} \mathbf{G}_{n} \mathbf{x}_{n}+\mathbf{n},
$$

where $\mathbf{G}_{n} \in \mathbb{C}^{\left(L_{\mathrm{x}}+L_{\mathrm{g}}-1\right) \times L_{\mathrm{x}}}$ is the convolutional matrix of $\mathbf{g}_{n}$. Again, for later investigations the load on the second hop is defined similar to (3) as

$$
\beta_{\mathrm{RD}}=M \cdot N \cdot L_{\mathrm{g}} \cdot R_{\mathrm{c}} .
$$

Hence, under the assumption of equally long channel impulse responses on both hops, the load on the second hop is $N$ times as large as on the first hop, i.e., $\beta_{\mathrm{RD}}=$ $N \cdot \beta_{\mathrm{SR}}$.

\section{Common detection schemes}

\subsection{Common IDM-STC detector}

In order to separate all $M \cdot N$ layers $\mathbf{x}_{m, n}$ at the destination, an iterative turbo detection is applied [8]. Figure 4 depicts the part of the overall detector for the detection of message $\mathbf{b}_{m}$ of source $S_{m}$. After soft-RAKE based interference cancelation (IC) with respect to all $M \cdot N$ layers, relay specific interleaving is reversed $\Pi_{\mathrm{r}, n}^{-1}$, yielding the LLRs $\Lambda_{\mathrm{c}_{m, n}^{\prime}}^{\mathrm{IC}}$. Since the relays transmitted over statistically independent channels $\mathbf{g}_{n}$, the LLRs $\Lambda_{\mathbf{c}_{m, n}^{\prime}}^{\mathrm{IC}}$ represent statistically independent observations of $\mathbf{c}_{m}^{\prime \prime}$ and, hence, are summed up.

$$
\mathbf{\Lambda}_{\mathbf{c}_{m}^{\prime}}^{\mathrm{IC}}=\sum_{n=1}^{N} \boldsymbol{\Lambda}_{\mathbf{c}_{m, n}^{\prime}}^{\mathrm{IC}}
$$

This de-interleaving and the subsequent summation (8) can be interpreted as the actual decoding of the dIDMSTC. The sum signal $\Lambda_{\mathrm{C}_{m}^{\prime}}^{\mathrm{IC}}$ is then de-interleaved using the user specific interleaver $\Pi_{m}^{-1}$, such that the de-interleaved LLRs for $\mathbf{c}_{m}$ are given by

$$
\Lambda_{\mathrm{c}_{m}}^{\mathrm{IC}}=\Pi_{m}^{-1}\left(\boldsymbol{\Lambda}_{\mathrm{c}_{m}^{\prime}}^{\mathrm{IC}}\right)=\Pi_{m}^{-1}\left(\sum_{n=1}^{N} \Pi_{\mathrm{r}, n}^{-1}\left(\boldsymbol{\Lambda}_{\mathrm{c}_{m, n}^{\prime \prime}}^{\mathrm{IC}}\right)\right) .
$$

Using $\Lambda_{\mathrm{C}_{m}}^{\mathrm{IC}}$, soft-input soft-output channel decoding $\mathcal{D}$ is performed. The decoding consists of the decoding of the repetition code $\mathcal{C}_{\text {rep }}$ which is a summation of the corresponding LLRs, followed by the decoding of the convolutional code $\mathcal{C}_{\text {conv }}$ using the well-known BCJR algorithm [19]. The BCJR delivers LLRs $\boldsymbol{\Lambda}_{\mathrm{b}_{m}}$ for the information bits $\mathbf{b}_{m}$ as well as LLRs $\boldsymbol{\Lambda}_{c_{m}}$ for the code bits $\mathbf{c}_{m}$. In order to obtain the extrinsic information generated by the decoder, the input LLRs $\Lambda_{\mathrm{C}_{m}}^{\mathrm{IC}}$ are subtracted from the output LLRs, yielding

$$
\boldsymbol{\Lambda}_{\mathrm{c}_{m}}^{\mathrm{ext}}=\boldsymbol{\Lambda}_{\mathrm{C}_{m}}-\boldsymbol{\Lambda}_{\mathrm{c}_{m}}^{\mathrm{IC}} \text {. }
$$

The extrinsic information $\boldsymbol{\Lambda}_{\mathrm{C}_{m}}^{\text {ext }}$ is then re-interleaved by the user specific interleaver $\Pi_{m}$ followed by the corresponding relay specific interleavers $\Pi_{r, n}$ and fed back as a-priori information to the IC. At the same time, the LLRs

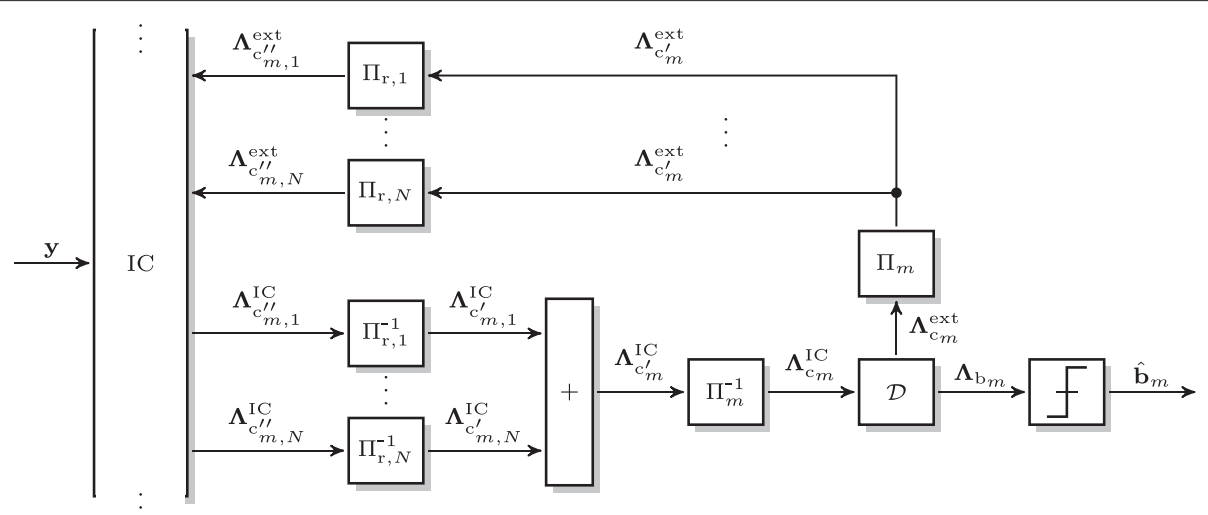

Figure 4 Structure of the common detection scheme at $D$ for dIDM-STC. Shown is the specific part for detection of the information word $\mathbf{b}_{m}$ of source $S_{m}$. 
$\boldsymbol{\Lambda}_{\mathrm{b}_{m}}$ of the information bits $\mathbf{b}_{m}$ are hard quantized, leading to the hard estimates $\hat{\mathbf{b}}_{m}=\mathcal{Q}\left(\boldsymbol{\Lambda}_{\mathrm{b}_{m}}\right)$. This iterative process is repeated until the CRC check of $\hat{\mathbf{b}}_{m}$ is correct or the maximum number of iterations $N_{\text {it }}$ is reached.

\subsubsection{Issues}

The major issue of the common detection scheme is that no information regarding the quality of the first hop transmission and the reliability of the decoding at the relays is taken into account. In contrast, it is inherently assumed that all detections at the relays are perfect, i.e., $\mathbf{b}_{m, n}=\mathbf{b}_{m}$, such that the first hop can be neglected for detection at the destination. Clearly, this assumption is not valid in practical systems as fading and noise lead to decoding errors at the relays which then propagate to the destination. The actual problems of the common detector with respect to erroneous decoding at the relays are twofold:

- The summation (9) of the de-interleaved LLRs is dominated by the strongest LLRs $\boldsymbol{\Lambda}_{\mathrm{c}_{m, n}^{\prime \prime}}^{\mathrm{IC}}$. This is especially a problem if one or more relays experience a weak channel on the first hop and a strong channel on the second hop, because the LLRs $\boldsymbol{\Lambda}_{\mathrm{c}_{m, n}^{\prime \prime}}^{\mathrm{IC}}$ only depend on the quality of the second hop but not on the first hop. Due to the weak first hop these relays are very likely to generate errors due to erroneous decoding. However, at the same time these relays dominate other relays with a weaker second hop which may have decoded correctly.

- The second issue is the loss of information about the individual relay signals $\mathbf{x}_{n}$ due to the summation (9) and the subsequent joint decoding. The extrinsic information $\boldsymbol{\Lambda}_{\mathrm{C}_{m}}^{\text {ext }}$ generated by the decoder is used as a-priori information for the next detection iteration for all relays. Thus, it is implicitly assumed that all relays transmitted the same codeword. If this is not the case, the feedback to the IC and the actual observations $\mathbf{y}$ are contradictory and the feedback may even degrade the performance of the IC. Hence, regarding the $\mathrm{IC}$, it would be favorable to process each relay separately during the iterative detection.

\subsection{Adaptive relaying}

One possibility to overcome the aforementioned problems is to adaptively select only the correctly decoded messages at each relay to be forwarded to the destination while all erroneously decoded messages are not transmitted [7]. That means, relay $R_{n}$ forwards only the messages of sources $S_{m}$ which were decoded correctly. The advantage of this strategy is that the common IDM-STC detection scheme could be applied with only marginal modifications to the system, e.g., the cyclic redundancy check $(\mathrm{CRC})$ code has to be exploited by the relays in order to determine the decoding success and to adaptively select only the correctly decoded messages.

However, even erroneous relays may contribute to the overall transmission as their relay information words $\mathbf{b}_{m, n}$, depending on the number of erroneous bits, may still be highly correlated to the source information words $\mathbf{b}_{m}$. Therefore, it seems not reasonable to discard erroneously decoded messages but to forward them anyway and let the destination handle the correct as well as the erroneous messages properly. Such a detection scheme is presented in the next section.

\section{Reliability-aware iterative detection (RAID)}

In order to consider decoding errors at the relays within the detector at the destination, a suitable model describing the overall transmission including the decoding reliabilities of the relays is required. On the one hand, this model should be accurate enough to actually improve the detection at the destination, on the other hand it should be simple enough to avoid an excessive increase in the complexity of the detector or in the signaling overhead.

\subsection{Equivalent transmission model for 1st hop transmission}

Based on the ideas presented in [14,15], decoding errors at the relays can be described using binary symmetric channels (BSCs) with a certain crossover probability. According to this description, the relay information word $\mathbf{b}_{m, n}$ in (4) is modeled as

$$
\mathbf{b}_{m, n}=\mathrm{BSC}_{m, n}\left\{\mathbf{b}_{m}, q_{m, n}\right\},
$$

where $q_{m, n}$ is the bit error probability of the estimate $\hat{\mathbf{b}}_{m}^{R_{n}}$ at relay $R_{n}$ regarding the source information word $\mathbf{b}_{m}$. This crossover probability is zero for perfect decoding at the relay and increases as the relay's decoding reliability decreases. Using this description, an equivalent transmission model for the transmission from the sources over the first hop to the relays, including decoding at the relays, can be derived. Figure 5 depicts such an equivalent joint model for the transmission of $\mathbf{b}_{m}$ via the relays $R_{1}$ up to $R_{N}$. The correlation between the source information word $\mathbf{b}_{m}$ and the relay information words $\mathbf{b}_{m, n}$ is described by $\mathrm{BSC}_{m, 1}$ up to $\mathrm{BSC}_{m, N}$ with error probabilities $q_{m, 1}$ up to $q_{m, N}$. These error probabilities $q_{m, n}$ are given by

$$
q_{m, n}=\frac{d_{\mathrm{H}}\left(\mathbf{b}_{m}, \hat{\mathbf{b}}_{m}^{R_{n}}\right)}{L_{\mathrm{b}}},
$$

where $d_{\mathrm{H}}(\cdot)$ denotes the Hamming distance and $L_{\mathrm{b}}$ is the length of the information sequence $\mathbf{b}_{m}$. Obviously, the calculation (12) would require perfect knowledge of $\mathbf{b}_{m}$ at the relays which is not the case in practical systems. However, an estimation of $q_{m, n}$ using the LLRs $\Lambda_{\mathrm{b}_{m}}^{R_{n}}$ of the 


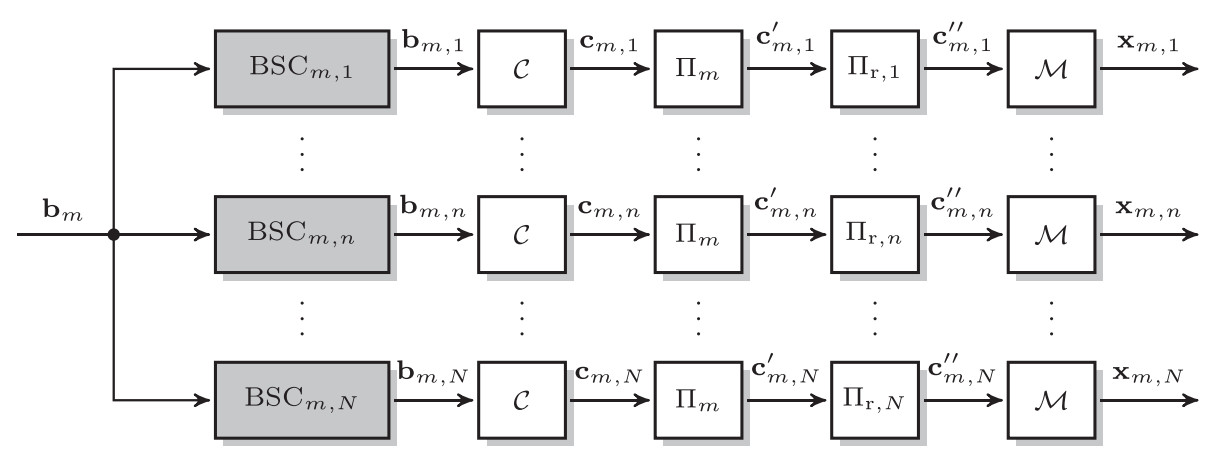

Figure 5 Equivalent transmission model for transmission of $\mathbf{b}_{\boldsymbol{m}}$ from $\boldsymbol{S}_{\boldsymbol{m}}$ via $\boldsymbol{R}_{\mathbf{1}}$ up to $\boldsymbol{R}_{\boldsymbol{N}}$ to $\boldsymbol{D}$. The shaded BSC blocks represent the shaded blocks from Figures 2 and 3.

information bits generated by the MUD at the relay is possible [20]. Denoting this estimate $\hat{q}_{m, n}$, it holds

$$
\hat{q}_{m, n}=\mathrm{E}\left\{\frac{1}{1+e^{\left|\Lambda_{\mathrm{b} m}^{R_{n}}\right|}}\right\} \approx \frac{1}{L_{\mathrm{b}}} \sum_{i=1}^{L_{\mathrm{b}}} \frac{1}{1+e^{\left|\Lambda_{\mathrm{b} m, i}^{R_{n} \mid}\right|}},
$$

where the expectation can be approximated by the time average due to the ergodic theorem. Note that $\hat{q}_{m, n} \neq 0$ even if the decoding at the relay was correct. Hence, in case of successful decoding at the relay ACK is signaled to the destination, while unsuccessful decoding leads to the signaling of a NAK in form of $\hat{q}_{m, n}$. The principle of this signaling is depicted in Figure 6, where $\mathrm{CRC}_{m, n}$ denotes the CRC check at relay $R_{n}$ regarding $\mathbf{b}_{m}$. For a more detailed discussion of the signaling refer to [16].

\subsection{RAID scheme}

Based on the presented equivalent transmission model, the new RAID is proposed. This detection scheme takes the decoding success $\left(\mathrm{CRC}_{m, n}=\mathrm{ACK} / \mathrm{NAK}\right)$ of the relays as well as the error probabilities $\hat{q}_{m, n}$ into account in order to improve the detection quality compared to the common detection scheme discussed in Section 3.1. In the following, the components of the RAID scheme, i.e., the relay grouping, the detection process and the weighted combining are discussed in detail.

\subsubsection{Relay grouping}

To address the second issue of the common detection strategy, i.e., the loss of information about the individual relay signals $\mathbf{x}_{m, n}$, a user specific separation of the correct relays and all erroneous relays is introduced. Since all correct relays have transmitted the same code word $\mathbf{c}_{m, n}=\mathbf{c}_{m}$, their LLRs can be combined after relay specific de-interleaving. All erroneous relays, however, may have transmitted pairwise different code words and, hence, are all processed separately.

Based on the decoding success $\left(\mathrm{CRC}_{m, n}=\mathrm{ACK} / \mathrm{NAK}\right)$ with respect to one specific source $S_{m}$, each relay $R_{n}$ is assigned to one of two disjoint groups, the group of relays which have correctly decoded the source message, i.e., $\mathbf{b}_{m, n}=\mathbf{b}_{m}$, and the group of relays which have not correctly decoded the source message, i.e., $\mathbf{b}_{m, n} \neq \mathbf{b}_{m}$. For the sake of notational simplicity, the set $\mathcal{R}_{m}$ of indices of the correct relays w.r.t. source $S_{m}$ and the set $\overline{\mathcal{R}}_{m}$ of indices of erroneous relays w.r.t. source $S_{m}$ are introduced

$$
\begin{aligned}
& \mathcal{R}_{m}=\left\{n \mid q_{m, n}=0,1 \leq n \leq N\right\} \\
& \overline{\mathcal{R}}_{m}=\left\{n \mid q_{m, n} \neq 0,1 \leq n \leq N\right\} .
\end{aligned}
$$

Obviously, the union of both sets is the set of the indices of all relays, i.e., $\mathcal{R}_{m} \cup \overline{\mathcal{R}}_{m}=\{1, \ldots, N\}$. Furthermore, two indexing functions $\rho_{m}$ and $\bar{\rho}_{m}$ are defined, such that

$$
\begin{aligned}
& \mathcal{R}_{m}=\left\{\rho_{m}(1), \ldots, \rho_{m}\left(I_{m}\right)\right\}, \quad I_{m}=\left|\mathcal{R}_{m}\right| \\
& \overline{\mathcal{R}}_{m}=\left\{\bar{\rho}_{m}(1), \ldots, \bar{\rho}_{m}\left(K_{m}\right)\right\}, \quad K_{m}=\left|\overline{\mathcal{R}}_{m}\right|
\end{aligned}
$$

with $\rho_{m}(1)<\rho_{m}(2)<\cdots<\rho_{m}\left(I_{m}\right)$ and $\bar{\rho}_{m}(1)<$ $\bar{\rho}_{m}(2)<\cdots<\bar{\rho}_{m}\left(K_{m}\right)$. This means $\rho_{m}(1)$ up to $\rho_{m}\left(I_{m}\right)$ represent the $I_{m}$ indices of the correct relays w.r.t $S_{m}$

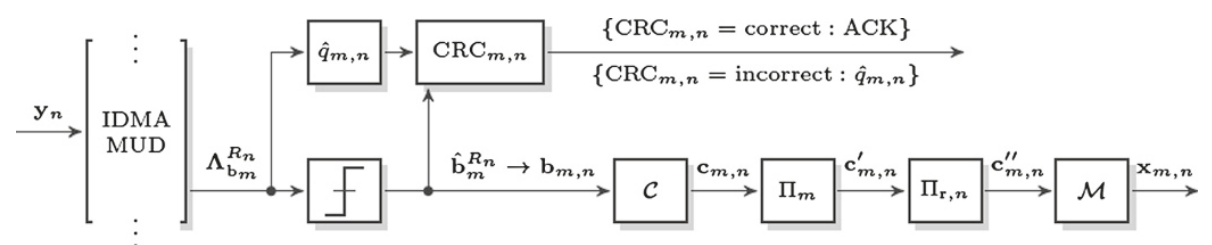

Figure 6 Principle of error estimation and signaling at relay $\boldsymbol{R}_{\boldsymbol{n}}$. Shown is the detection and error estimation for $\mathbf{b}_{m}$. 
and $\bar{\rho}_{m}(1)$ up to $\bar{\rho}_{m}\left(K_{m}\right)$ represent the $K_{m}$ indices of the erroneous relays w.r.t $S_{m}$, i.e.,

$$
\begin{aligned}
\mathbf{b}_{m, \rho_{m}(i)}=\mathbf{b}_{m}, & 1 \leq i \leq I_{m} \\
\mathbf{b}_{m, \bar{\rho}_{m}(k)} \neq \mathbf{b}_{m}, & 1 \leq k \leq K_{m} .
\end{aligned}
$$

Figure 7 shows the part of the overall proposed detector which is relevant to the detection of $\mathbf{b}_{m}$. The LLRs from the IC are grouped based on the decoding success $(\mathrm{ACK} / \mathrm{NAK})$ at the relays. Since the correct relays have transmitted the same code word $\mathbf{c}_{m}$, their LLRs are summed up after relay specific de-interleaving, similar to the common detection scheme, i.e.,

$$
\boldsymbol{\Lambda}_{\mathrm{c}_{m, \rho_{m}}^{\prime}}^{\mathrm{IC}}=\sum_{i=1}^{I_{m}} \Pi_{\mathrm{r}, \rho_{m}(i)}^{-1}\left(\boldsymbol{\Lambda}_{\mathrm{c}_{m, \rho_{m}(i)}^{\prime \prime}}^{\mathrm{IC}}\right)
$$

and are then jointly de-interleaved by the user specific interleaver and jointly decoded (bottom part). The erroneous relays, however, have transmitted different code words and are, therefore, processed and decoded separately (top part). The goal of this first stage of the detection is the best possible estimation of the relay information words $\mathbf{b}_{m, n}$ and not of the source information words $\mathbf{b}_{m}$. The estimation of the source information words is exclusively performed in the second stage of the detector.
Finally, after the last iteration, the $K_{m}+1$ decoders $\mathcal{D}$ deliver LLRs $\boldsymbol{\Lambda}_{\mathrm{b}_{m, \rho_{m}}}$ for the information words of the correct relays and LLRs $\Lambda_{\mathrm{b}_{m, \bar{\rho}(k)}}$ for the $K_{m}$ information words of the erroneous relays. The explicit decoding, hard decision and subsequent re-encoding at the relays ensures that all relays actually transmitted a valid code word which is fundamental for the validity of the joint equivalent transmission model.

\subsubsection{Weighted combining}

Based on the estimates for the relay information words $\mathbf{b}_{m, n}$, now an overall estimate for the source information word $\mathbf{b}_{m}$ should be determined. This estimate should not only include the LLRs from the correct relays, but also the LLRs from the erroneous relays as, depending on the error probabilities $\hat{q}_{m, n}$, the relay information of the erroneous relays is still correlated to the source information.

The question arises, how to obtain an estimate $\Lambda_{\mathrm{b}_{m}}^{n}$ for the source information $\mathbf{b}_{m}$ given the estimate $\boldsymbol{\Lambda}_{\mathrm{b}_{m, n}}$ for a specific relay information word $\mathbf{b}_{m, n}$ and the corresponding error probability $\hat{q}_{m, n}$. How does the BSC modeling of the first hop transmission translate to the LLRs for the relay information word and the source information word? Figure 8 illustrates this relationship, where the function $\mathcal{W}(\cdot)$ needs to be found, such that

$$
\boldsymbol{\Lambda}_{\mathrm{b}_{m}}^{n}=\mathcal{W}\left(\boldsymbol{\Lambda}_{\mathrm{b}_{m, n}}, \hat{q}_{m, n}\right)
$$

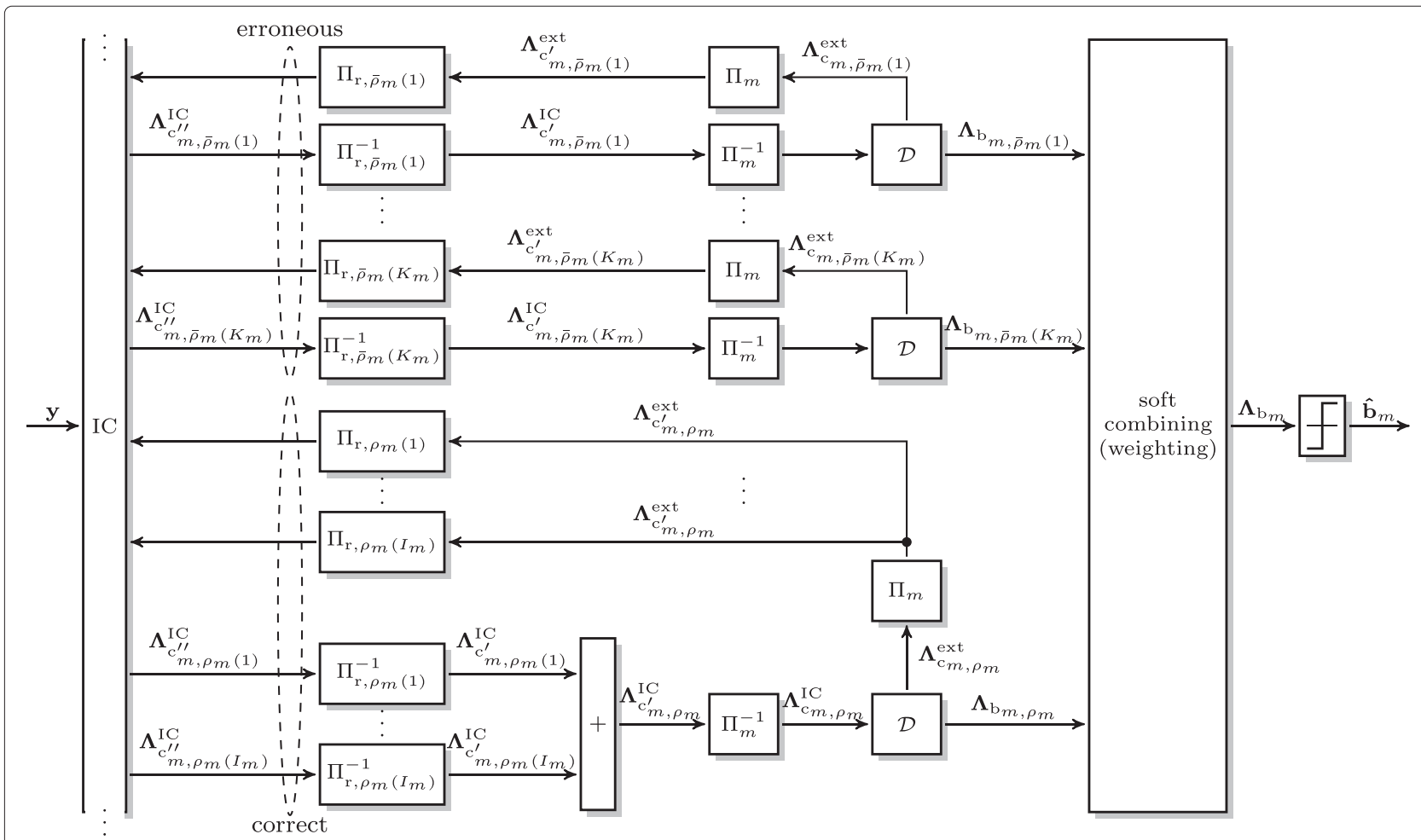

Figure 7 Structure of the proposed RAID scheme at $D$ for dIDM-STC. Shown is the specific part for detection of the information word $\mathbf{b}_{m}$ of source $S_{m}$. 


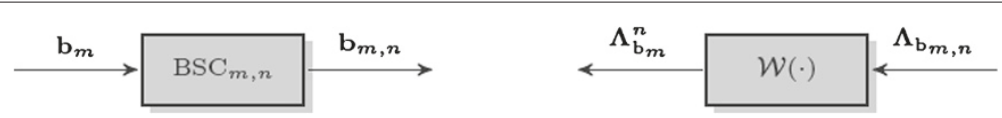

Figure 8 BSC modeling of relay information word $\mathbf{b}_{m, n}$ and corresponding weighting function $\mathcal{W}(\cdot)$ for its LLRs $\Lambda_{\mathbf{b}_{m, n}}$.

with $\boldsymbol{\Lambda}_{\mathrm{b}_{m}}^{n}$ denoting the estimate for the source information $\mathbf{b}_{m}$ taking only relay $R_{n}$ into account. In order to find this function, the estimate $\boldsymbol{\Lambda}_{\mathrm{b}_{m}}^{n}$ for an arbitrary element $b_{m}$ of $\mathbf{b}_{m}$ is written as

$$
\begin{aligned}
\Lambda_{\mathrm{b}_{m}}^{n} & =\log \left(\frac{p\left(b_{m}=0, y\right)}{p\left(b_{m}=1, y\right)}\right) \\
& =\log \left(\frac{P\left(b_{m}=0 \mid y\right) p(y)}{P\left(b_{m}=1 \mid y\right) p(y)}\right) \\
& =\log \left(\frac{P\left(b_{m}=0 \mid y\right)}{P\left(b_{m}=1 \mid y\right)}\right) .
\end{aligned}
$$

By using the law of total probabilities [21], the probabilities of the source information $b_{m}$ can be written w.r.t. the probabilities of the relay information $b_{m, n}$ as

$$
\begin{aligned}
P\left(b_{m}=0 \mid y\right) & =P\left(b_{m}=0 \mid b_{m, n}=0, y\right) P\left(b_{m, n}=0 \mid y\right) \\
& +P\left(b_{m}=0 \mid b_{m, n}=1, y\right) P\left(b_{m, n}=1 \mid y\right) \\
P\left(b_{m}=1 \mid y\right) & =P\left(b_{m}=1 \mid b_{m, n}=1, y\right) P\left(b_{m, n}=1 \mid y\right) \\
& +P\left(b_{m}=1 \mid b_{m, n}=0, y\right) P\left(b_{m, n}=0 \mid y\right) .
\end{aligned}
$$

The probabilities of $b_{m}$ given $b_{m, n}$ solely depend on the crossover probability $\hat{q}_{m, n}$ of the BSC,

$$
\begin{aligned}
P\left(b_{m}=0 \mid b_{m, n}=0\right) & =P\left(b_{m}=1 \mid b_{m, n}=1\right) \\
& =1-\hat{q}_{m, n} \\
P\left(b_{m}=1 \mid b_{m, n}=0\right) & =P\left(b_{m}=0 \mid b_{m, n}=1\right) \\
& =\hat{q}_{m, n}
\end{aligned}
$$

such that the estimate $\Lambda_{\mathrm{b}_{m}}^{n}$ can be rewritten as (25a). Expressing the probabilities by LLRs [22]

$$
\begin{aligned}
& P\left(b_{m, n}=0 \mid y\right)=\frac{e^{\boldsymbol{\Lambda}_{\mathrm{b}_{m, n}}}}{1+e^{\boldsymbol{\Lambda}_{\mathrm{b}_{m, n}}}} \\
& P\left(b_{m, n}=1 \mid y\right)=\frac{1}{1+e^{\boldsymbol{\Lambda}_{\mathrm{b}_{m, n}}}}
\end{aligned}
$$

leads to (25b) and after some algebraic manipulations to (25c). Thus, the desired function $\mathcal{W}(\cdot)$ is finally found to be

$$
\begin{aligned}
& \boldsymbol{\Lambda}_{\mathrm{b}_{m}}^{n}=\log \left(\frac{P\left(b_{m, n}=0 \mid y\right)\left(1-\hat{q}_{m, n}\right)+P\left(b_{m, n}=1 \mid y\right) \hat{q}_{m, n}}{P\left(b_{m, n}=1 \mid y\right)\left(1-\hat{q}_{m, n}\right)+P\left(b_{m, n}=0 \mid y\right) \hat{q}_{m, n}}\right) \\
& =\log \left(\frac{\frac{e^{\Lambda_{b_{m, n}}}}{1+e^{\Lambda_{b_{m, n}}}}\left(1-\hat{q}_{m, n}\right)+\frac{1}{1+e^{\Lambda_{b_{m, n}}}} \hat{q}_{m, n}}{\frac{1}{1+e^{\Lambda_{b_{m, n}}}}\left(1-\hat{q}_{m, n}\right)+\frac{e^{\Lambda_{b_{m, n}}}}{1+e^{\Lambda_{b_{m, n}}}} \hat{q}_{m, n}}\right)
\end{aligned}
$$

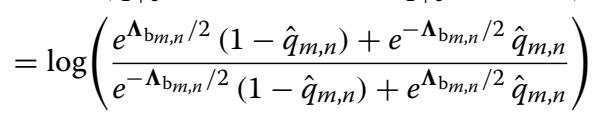

$$
\begin{aligned}
& \mathcal{W}\left(\boldsymbol{\Lambda}_{\mathrm{b}_{m, n}}, \hat{q}_{m, n}\right) \\
& =\log \left(\frac{e^{\boldsymbol{\Lambda}_{\mathrm{b}_{m, n}} / 2}\left(1-\hat{q}_{m, n}\right)+e^{-\boldsymbol{\Lambda}_{\mathrm{b}_{m, n}} / 2} \hat{q}_{m, n}}{e^{-\boldsymbol{\Lambda}_{\mathrm{b} m, n} / 2}\left(1-\hat{q}_{m, n}\right)+e^{\boldsymbol{\Lambda}_{\mathrm{b} m, n} / 2}} \hat{q}_{m, n}\right),
\end{aligned}
$$

where $\mathcal{W}\left(\boldsymbol{\Lambda}_{\mathrm{b}_{m, n}}, \hat{q}_{m, n}\right)$ is a shorthand notation for applying (25c) to every element of $\boldsymbol{\Lambda}_{\mathrm{b}_{m, n}}$.

In (25c) the error probability $\hat{q}_{m, n}$ of the BSC obviously leads to a weighting of the estimates of the relay information word given by $\boldsymbol{\Lambda}_{\mathrm{b}_{m, n}}$. For completely uncorrelated $\mathbf{b}_{m, n}$ and $\mathbf{b}_{m}$, i.e., $\hat{q}_{m, n}=0.5$, the relay transmitted no information regarding $\mathbf{b}_{m}$ and, hence, $\boldsymbol{\Lambda}_{\mathrm{b}_{m}}^{n}=0$. However, as $\hat{q}_{m, n}$ decreases, $\boldsymbol{\Lambda}_{\mathrm{b}_{m}}^{n}$ tends to $\boldsymbol{\Lambda}_{\mathrm{b}_{m, n}}$ giving an estimate of $\mathbf{b}_{m}$ with respect to the information from relay $R_{n}$.

Since all transmit channels are statistically independent, the observations from all relays can be summed up resulting in the estimate

$$
\begin{aligned}
\boldsymbol{\Lambda}_{\mathrm{b}_{m}} & =\boldsymbol{\Lambda}_{\mathrm{b}_{m, \rho_{m}}}+\sum_{k=1}^{K_{m}} \mathcal{W}\left(\boldsymbol{\Lambda}_{\mathrm{b}_{m}, \bar{\rho}_{m}(k)}, \hat{q}_{m, n}\right) \\
& =\boldsymbol{\Lambda}_{\mathrm{b}_{m, \rho_{m}}}+\sum_{k=1}^{K_{m}} \boldsymbol{\Lambda}_{\mathrm{b}_{m}}^{\bar{\rho}_{m}(k)}
\end{aligned}
$$

where $\boldsymbol{\Lambda}_{\mathrm{b}_{m, \rho_{m}}}$ is the unweighted estimate from the correct relays. Finally, hard quantization leads to the overall estimate $\hat{\mathbf{b}}_{m}=\mathcal{Q}\left(\boldsymbol{\Lambda}_{\mathrm{b}_{m}}\right)$ for the source message $\mathbf{b}_{m}$.

\subsection{Pseudo code}

In order to facilitate the comprehension of the proposed RAID scheme, a pseudo code for the overall detection process is given in Algorithm 1. The algorithm requires the set of all CRC check results $\left\{\mathrm{CRC}_{m, n}\right\}$, the set of all error probabilities $\left\{\hat{q}_{m, n}\right\}$, the set of all channel impulse 


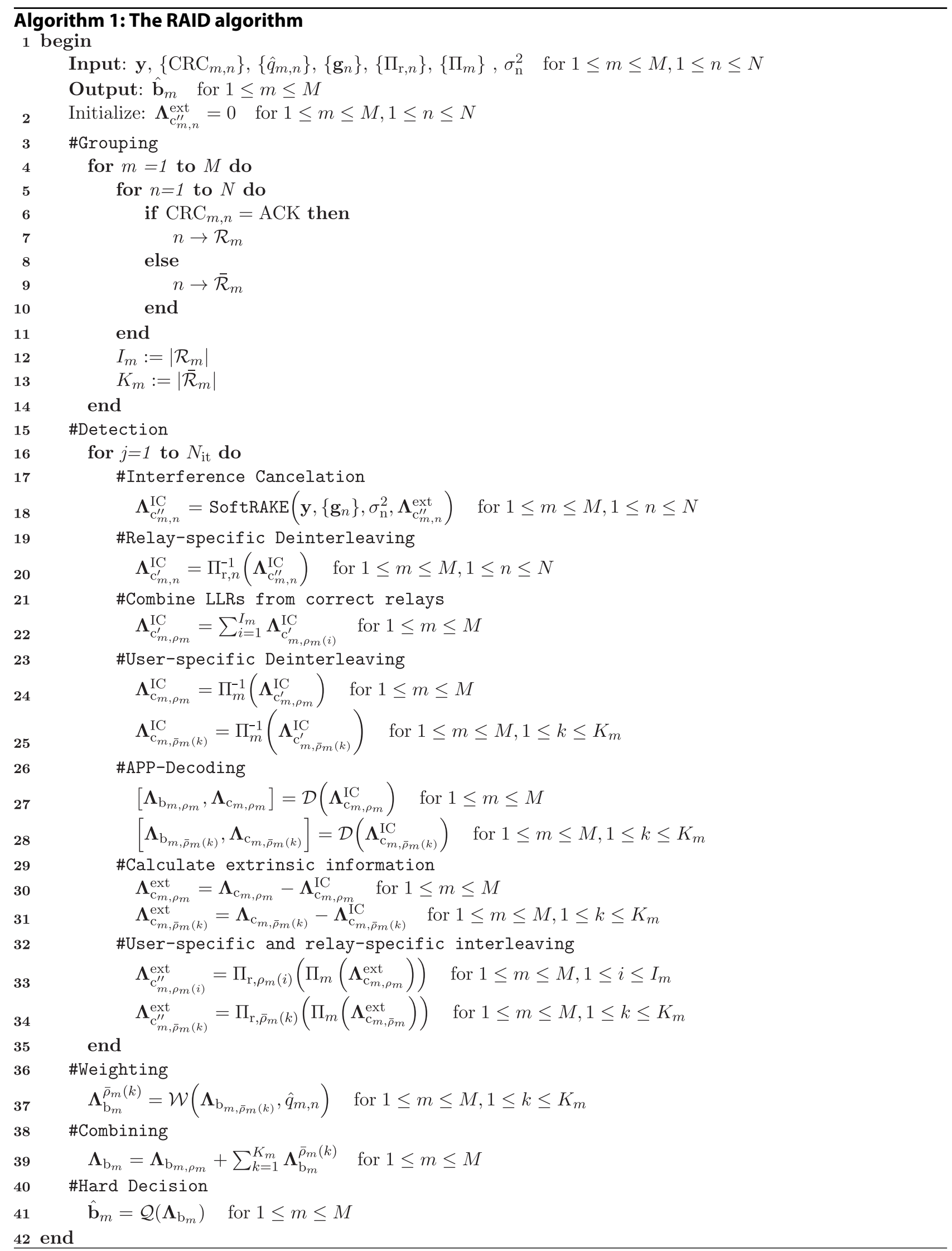


responses from the relays to the destination $\left\{\mathbf{g}_{n}\right\}$, the sets of all relay specific and user specific interleavers $\left\{\Pi_{\mathrm{r}, n}\right\}$ and $\left\{\Pi_{n}\right\}$ and the noise variance $\sigma_{\mathrm{n}}^{2}$.

After the grouping, as described in Section 4.2.1 (lines $3-14$ ), the iterative detection process is performed (lines 15-35). Besides some interleaving and de-interleaving operations, it mainly consists of the softRAKE detection (line 18), the combining of the information from the correct relays (line 22) and the APP-decoding (lines 27-28). Finally, after the detection, the weighting (line 37) and combining (line 39), as described in Section 4.2.2, is performed. The hard decision (line 41) leads to the hard estimates of the user information words $\hat{\mathbf{b}}_{m}$.

\subsection{Computational complexity}

The computational complexity of the presented detection schemes, i.e., $\mathrm{cDF}, \mathrm{aDF}$, and RAID, mainly differs in the number of APP-decodings per frame. Hence, the number of APP-decodings per frame is a useful measure in order the compare the complexity of the schemes. Table 1 gives an overview of the number of decodings per frame. As can be seen, for $\mathrm{cDF}$ and $\mathrm{aDF}$ one APP-decoding per user, frame and iteration is required, independent of the number of successful relays. For RAID, however, the number of APP-decodings depends on the number of successful relays. In the best case, i.e., all relays were successful, only one decoding operation per iteration and user is required, leading to the same complexity as $\mathrm{CDF}$ and $\mathrm{aDF}$. In the worst case, every relay message is decoded separately, resulting in a complexity $N$ times as high as for $\mathrm{cDF}$ and aDF. Note that the aDF scheme would fail in the worst case scenario, while cDF and RAID might still achieve correct decoding.

\section{Numerical results}

For numerical investigations, a two-hop relay system with $M$ sources $S_{m}, N=4$ parallel relays $R_{n}$ and one common destination $D$ as depicted in Figure 1 is considered. The distance between the central source and the destination is normalized to $d_{\mathrm{SD}}=1$ and the inter-relay distance is set so $d_{\mathrm{R}}=0.2$. Frequency-selective block Rayleigh fading with $L=L_{\mathrm{h}}=L_{\mathrm{g}}$ i.i.d. channel taps is assumed on

\section{Table 1 Comparison of the computational complexity of} CDF, aDF, and RAID

\begin{tabular}{lccc}
\hline & $\begin{array}{c}\text { Number of APP-Decodings } \\
\text { per frame }\end{array}$ & Best case & Worst case \\
\hline CDF & $N_{\text {it }} M$ & $N_{\text {it }} M$ & $N_{\text {it }} M$ \\
aDF & $N_{\text {it }} M$ & $N_{\text {it }} M$ & $N_{\text {it }} M$ \\
RAID & $N_{\text {it }} \sum_{m=1}^{M} \min \left\{1+\left|\overline{\mathcal{R}}_{m}\right|, N\right\}$ & $N_{\text {it }} M$ & $N_{\text {it }} M N$ \\
\hline
\end{tabular}

$M$ number of users, $N$ number of relays, $\left|\mathcal{R}_{m}\right|$ number of erroneous relays w.r.t. $S_{m}$ and $N_{i t}$ number of iterations per frame. both hops and the path loss exponent is set to $\epsilon=3$. For channel coding, a combination of the non-recursive half-rate $(5,7)_{8}$ convolutional code and a repetition code of rate $R_{\mathrm{c} \text {,rep }}$ is applied and the codeword length is set to $L_{\mathrm{c}}=1024$ codebits. The QPSK alphabet $\mathcal{A}$ with $\sigma_{\mathrm{x}}^{2}=1$ is chosen and the relay transmit signals are normalized such that $\sigma_{\mathbf{x}_{n}}^{2}=1$ regardless of the number of supported users $M$. That way, all schemes have the same power cost for a given number of users $M$, making the comparisons fair. For detection at the relays and at the destination $N_{\text {it }}=10$ iterations are performed. Initially, the error probabilities $q_{m, n}$ are assumed to be perfectly known at the destination.

\subsection{Single user}

First, a single user system is considered, i.e., $M=1$. Figure 9 depicts the frame error rate (FER) at the destination for the common detection scheme (cDF) and the proposed RAID scheme for different code rates $R_{\mathrm{c}, \text { rep }}$ over flat $(L=1$, solid) and frequency selective channels $(L=4$, dashed). As reference, also the adaptive relay system is given $(\mathrm{aDF})$. Starting with the system without repetition coding, i.e., $R_{\mathrm{c}, \text { rep }}=1$, it can be seen, that the RAID scheme clearly outperforms the common scheme for flat, as well as for frequency selective channels. The adaptive scheme, however, performs significantly better than the RAID scheme. Also, all three detection schemes lead to an error floor for frequency selective channels.

Since soft-RAKE-detection is applied, overall $N \cdot L$ layers have to be separated at the destination. In case of $L=4$ this corresponds to a load of $\beta_{\mathrm{RD}}=8$. Hence, in this scenario, the system is eight times overloaded. Clearly, a separation of all layers fails at this high load even for high signal-to-noise ratios (SNRs) resulting in the observed error floor. Even for flat fading the system is significantly overloaded, i.e., $\beta_{\mathrm{RD}}=2$. In this case, the adaptive scheme outperforms the RAID scheme, since the system is interference limited and adaptively switching erroneous relays off decreases the load and leads to a better separation of the layers at the destination.

Introducing a repetition code of rate $R_{\mathrm{c}, \text { rep }}=\frac{1}{2}$, as depicted in Figure 9b, reduces the system load to $\beta_{\mathrm{RD}}=1$ for the flat fading case and $\beta_{\mathrm{RD}}=4$ for the frequency selective fading case. The RAID as well as the adaptive scheme now perform significantly better than the common scheme. As can be seen, the advantage of the adaptive scheme has vanished. For flat fading the RAID scheme even performs slightly better than the adaptive scheme. In this case, all layers can be successfully separated by the IC. However, while the adaptive scheme does not exploit erroneous relays, the proposed RAID scheme leads to a slightly better performance by also taking these relays into account. For the frequency selective channels, however, the load is still high, and the adaptive scheme leads to a marginally better performance than RAID. 


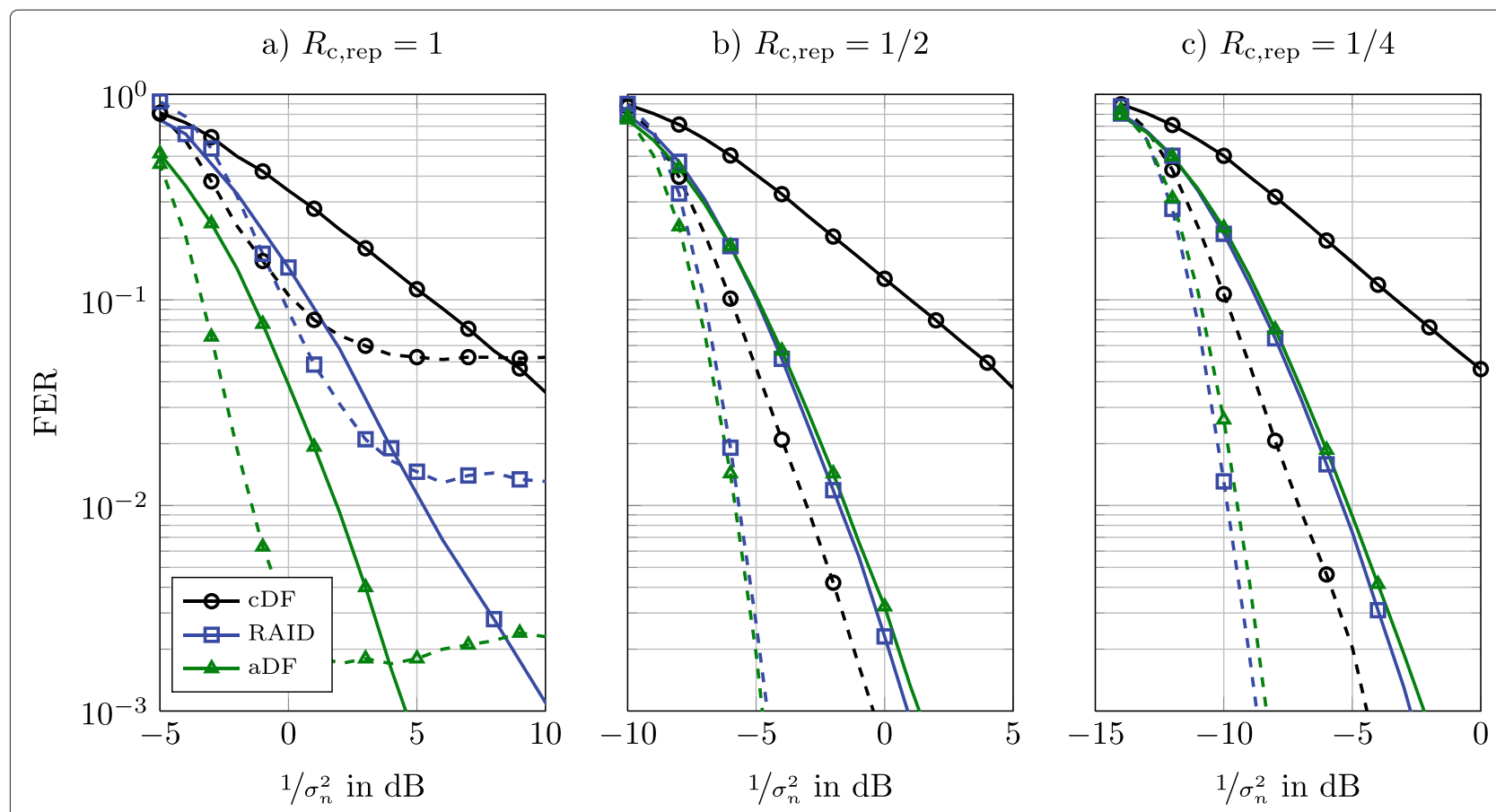

Figure 9 FER at the destination for common detection, RAID and adaptive relaying and different coderates $R_{\mathrm{c}, \text { rep }}$ of the repetition code. $M=1$ user. Solid: $L=1$ channel taps, dashed: $L=4$ channel taps.

Further decreasing the code rate of the repetition code to $R_{\mathrm{c}, \text { rep }}=\frac{1}{4}$, as in Figure $9 \mathrm{c}$, reduces the load to $\beta_{\mathrm{RD}}=\frac{1}{2}$ for flat fading and $\beta_{\mathrm{RD}}=2$ for frequency selective fading. Since the system is not interference limited anymore, this results in a better performance for RAID compared to the adaptive scheme even for the frequency selective case.

In Figure 10, the corresponding end-to-end throughput $\eta$, assuming a selective repeat automatic repeat request (ARQ) protocol, is shown. For the given system parameters, i.e., QPSK modulation and a half-rate convolutional code, it reads

$$
\begin{aligned}
\eta & =\log _{2}(|\mathcal{A}|) \cdot R_{\mathrm{c}, \mathrm{conv}} \cdot R_{\mathrm{c}, \text { rep }} \cdot(1-\mathrm{FER}) \\
& =R_{\mathrm{c}, \text { rep }} \cdot(1-\mathrm{FER})
\end{aligned}
$$

where $|\mathcal{A}|$ denotes the cardinality of $\mathcal{A}$. While the system without repetition coding clearly leads to the worst performance in terms of FER for all three schemes, it outperforms the other systems in terms of throughput above approx. $\frac{1}{\sigma_{\mathrm{n}}^{2}}=-5 \mathrm{~dB}$ for aDF and $\frac{1}{\sigma_{\mathrm{n}}^{2}}=-3 \mathrm{~dB}$ to $\frac{1}{\sigma_{\mathrm{n}}^{2}}=-2 \mathrm{~dB}$ for RAID and cDF. In the high SNR region the throughput clearly tends to the spectral efficiency $R_{\mathrm{c} \text {,rep }}$ of the system. Interestingly, for $R_{\mathrm{c}, \text { rep }}=1$ and $L=4$ a) $L=1$

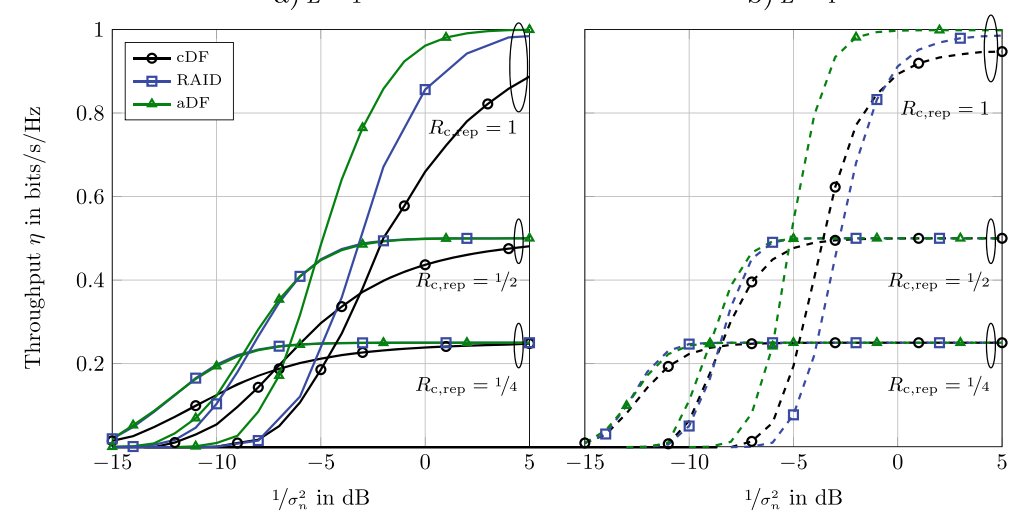

Figure 10 Achieved end-to-end throughput for all strategies and different coderates $\boldsymbol{R}_{\mathrm{c}, \text { rep }}$ of the repetition code. $M=1$ user. 
the common scheme achieves a higher throughput than RAID up to $\frac{1}{\sigma_{\mathrm{n}}^{2}}=-1 \mathrm{~dB}$. Again, the reason for this behavior is the high load in the system and the very low SNR per layer the detector experiences at this point. The LLRs delivered by the IC are, hence, very small. In this case, it is better to add all available LLRs up, trying to increase the signal level and achieve successful joint decoding than to separate the layers according to the decoding success of the relays and thereby keeping the signal levels low. Above $\frac{1}{\sigma_{\mathrm{n}}^{2}}=-1 \mathrm{~dB}$, the signal levels are sufficiently high and the RAID outperforms the common scheme. The same behavior can be seen for $R_{\mathrm{c} \text {,rep }}=\frac{1}{2}$ and $L=4$ but with much smaller characteristic. This is due to the decoding of the repetition code, which corresponds to the summation of two subsequent LLRs. The signal levels after IC are hereby increased sufficiently and the RAID achieves almost the same performance as cDF in the lower SNR region up to approx. $\frac{1}{\sigma_{\mathrm{n}}^{2}}=-8 \mathrm{~dB}$. Above this SNR, again RAID clearly outperforms cDF. Finally, for $R_{\mathrm{c}, \text { rep }}=\frac{1}{4}$ RAID always performs better than $\mathrm{CDF}$, as the observed behavior is completely avoided for higher code rates $R_{\mathrm{c}, \text { rep. }}$.

\subsection{Estimation of error probability $q_{m, n}$}

While the previously shown results for the RAID scheme were all based on perfect knowledge of $q_{m, n}$, subsequently the impact of the usage of its estimation $\hat{q}_{m, n}$ given in (13) is investigated. Figure 11 shows samples of the bit error probability $q_{m, n}$ and its estimate $\hat{q}_{m, n}$ for different frame sizes $L_{\mathrm{b}}$ in a double logarithmic representation. These samples were obtained by Monte Carlo simulations, i.e., for transmissions at varying SNR the resulting $q_{m, n}$ and the corresponding $\hat{q}_{m, n}$ were calculated and plotted. The solid line indicates equality, while a mark above the line means a too high and a mark below the line a too low estimate. First, in the plots the quantized nature of $q_{m, n}$ can be seen. Since only integer numbers of erroneous bits can occur, $q_{m, n}$ is limited to the values $\frac{1}{L_{\mathrm{b}}}, \frac{2}{L_{\mathrm{b}}}, \ldots$ For $L_{\mathrm{b}}=64$, e.g., this is $0.0156,0.0312, \ldots$, which corresponds to the marks in the plot. Clearly, for larger frame lengths $L_{\mathrm{b}}$, lower error probabilities are possible, e.g., $\frac{1}{256}=0.0039$ for $L_{\mathrm{b}}=256$ and $\frac{1}{2560}=0.004$ for $L_{\mathrm{b}}=2560$. In addition, the size of the quantization steps gets smaller, as it is also given by $\frac{1}{L_{\mathrm{b}}}$. On the other hand, the estimate $\hat{q}_{m, n}$ is continuously distributed as the LLRs $\Lambda_{\mathrm{b}_{m}}^{R_{n}}$ the estimate is based on, are continuously distributed.

Apparently, also the quality of the estimate strongly depends on the frame size. While for a frame size of $L_{\mathrm{b}}=64$ information bits the estimate already significantly deviates from the true value for error rates of $10^{-1}$, the estimate improves for larger frame lengths. The reason for this behavior is the approximation in (13). Since the expectation can only be estimated using the mean over all LLRs within one frame, the estimate clearly improves as the frame length increases.

Another interesting observation is the behavior of the estimates $\hat{q}_{m, n}$ for small error probabilities. The lower the error probability $q_{m, n}$ is, the stronger deviates the estimate

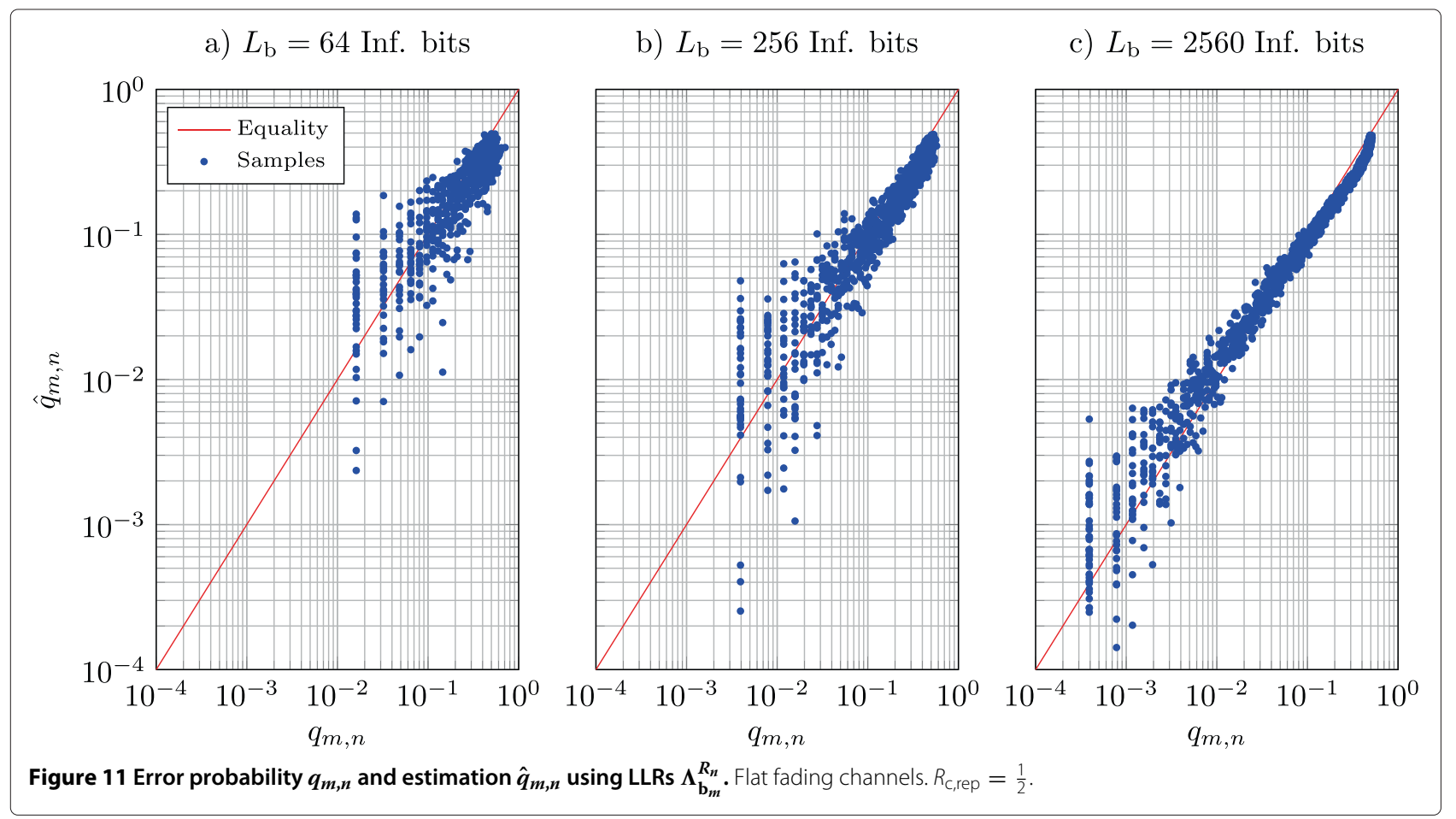


$\hat{q}_{m, n}$ from $q_{m, n}$. Again, the reason for this is the approximation in (13). Strictly speaking, the expectation has to be calculated with respect to the overall distribution of $\Lambda_{\mathrm{b}_{m}}^{R_{n}}$, i.e., all events which lead to one specific $q_{m, n}$ need to be taken into account in order to obtain a valid estimation. However, since only one frame with a specific channel realization is available at the relay, the influences of the channel are neglected in the approximation of the estimate, leading to the observed deviation for single events. Clearly, this is also true for larger error rates $q_{m, n}$. However, due to the logarithmic representation, the relative deviation is much smaller.

Finally, it can be seen from Figure 11, that for very high error probabilities, (13) leads to an underestimation, while for lower error probabilities $q_{m, n}$ is slightly overestimated. This leads to an overall more conservative behavior of the system due to the weighting (27).

In Figure 12, the achieved FERs at the destination using RAID with $q_{m, n}$ or its estimate $\hat{q}_{m, n}$ are depicted. Figure 12a) shows the FERs for both cases for flat $(L=$ 1 , solid) and frequency selective ( $L=4$, dashed) fading channels. While both methods lead to very similar results, using the estimate $\hat{q}_{m, n}$ instead of $q_{m, n}$ results in a small performance degradation. In Figure $12 \mathrm{~b}$, the same results are plotted again using a double logarithmic representation, i.e., each mark denotes the achieved FER for both methods at one specific SNR. All marks tend to lay above the equality line, indicating slightly higher FERs using the estimate $\hat{q}_{m, n}$ compared to $q_{m, n}$. In Figure 12c) the same comparison is drawn using frames of $L_{\mathrm{b}}=256$ information bits, corresponding to $L_{\mathrm{c}}=1024$ code bits. Obviously, although the estimate $\hat{q}_{m, n}$ still significantly deviates from the true $q_{m, n}$ for this frame size, the estimation (13) is sufficiently accurate as both methods lead to almost exactly the same error rates at the destination.

\subsection{Multiple users}

Finally, the performance evaluations are extended to multi-user scenarios. Specifically, the given topology is extended to $M=2$ and $M=4$ sources, respectively, with an inter-source spacing of $d_{\mathrm{S}}=0.1$. Figure 13 shows the results for a system with a repetition code of rate $R_{\mathrm{c}, \text { rep }}=\frac{1}{4}$. As a benchmark, the FERs at the destination for genie relays are given, i.e., the relays always decode perfectly and errors only occur on the second hop. For the genie relays, an increase of number of sources $M$ from 1 to 2 and from 2 to 4 , respectively, basically results in a SNR loss of $3 \mathrm{~dB}$ as the relay power is independent of the number of supported users $M$ and, hence, a doubling of the number of users $M$ leads to a halving of the power per user and relay. For $L=4$ and $M=4$ users, as depicted in Figure 13b, an error floor due to the high load of $\beta_{\mathrm{RD}}=M \cdot N \cdot L \cdot R_{\mathrm{c}}=8$ can be observed. For $\mathrm{cDF}$ and RAID the gaps between the curves for different user numbers are significantly smaller than $3 \mathrm{~dB}$. This indicates that the overall performance is primarily dominated by the performance on the first hop and, hence, by the decoding at the relays. In fact, for $\mathrm{cDF}$ and $L=1$ a typical IDMA behavior [10] as applied on the first hop, can be observed. With increasing system load the performance

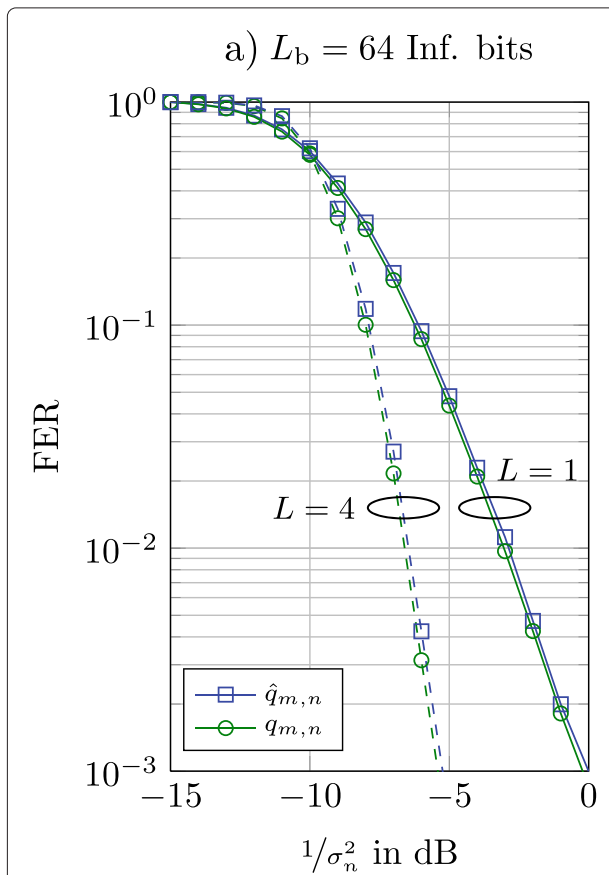

b) $L_{\mathrm{b}}=64$ Inf. bits

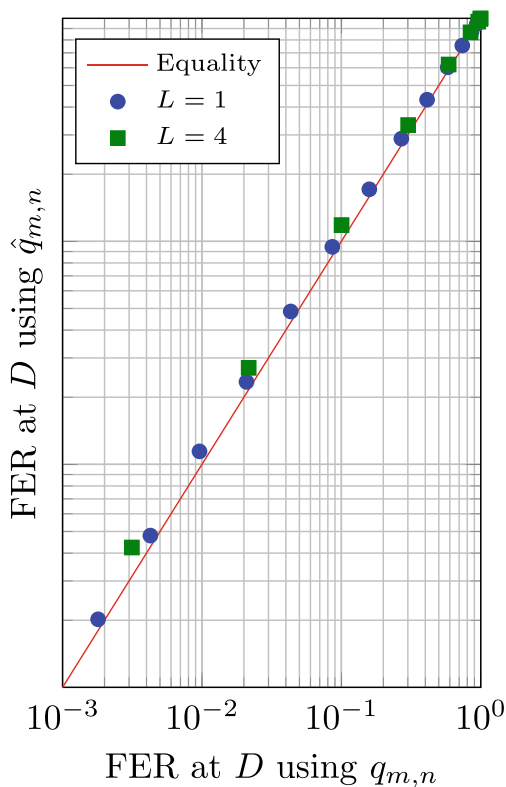

c) $L_{\mathrm{b}}=256$ Inf. bits

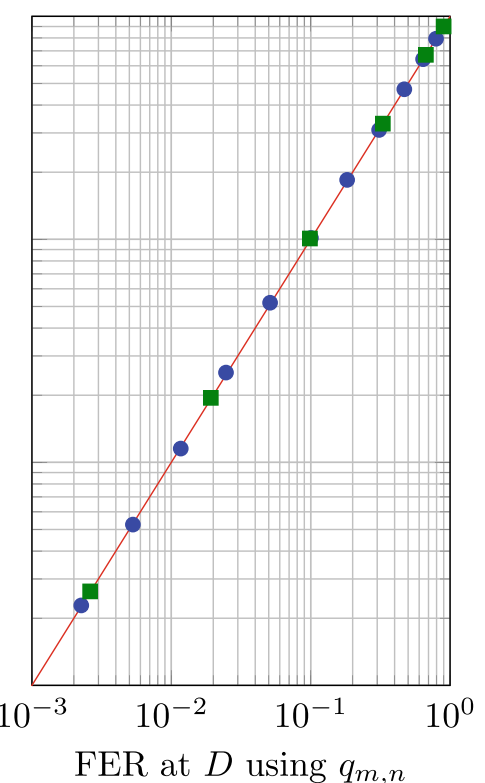

Figure 12 FER at destination using $\boldsymbol{q}_{\boldsymbol{m}, \boldsymbol{n}}$ and estimate $\hat{\boldsymbol{q}}_{\boldsymbol{m}, \boldsymbol{n}}$ for RAID. $R_{\mathrm{c} \text {, rep }}=\frac{1}{2}$. 
a) $L=1$

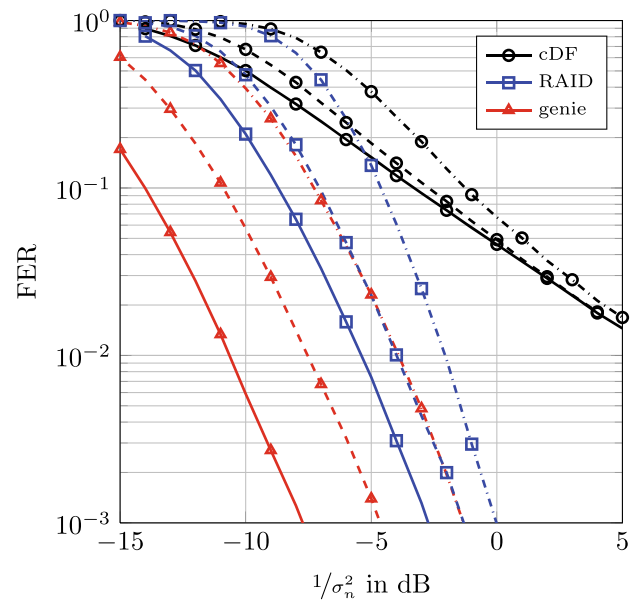

b) $L=4$



Figure 13 FER at destination for RAID and CDF for different numbers of users $M=1$ (solid), $M=2$ (dashed), $M=4$ (dashdotted).

$R_{\text {c, rep }}=\frac{1}{4}$.

of the system gets worse but still converges to the single user bound above a certain SNR threshold.

In Figure 14, a non-overloaded multi-user system with a repetition code of rate $R_{\mathrm{c}, \text { rep }}=\frac{1}{32}$ is given. Principally, for this system the same observations as for the system in Figure 13 can be made. However, even for $M=4$ users and frequency selective fading $(L=4)$, corresponding to a load of $\beta_{\mathrm{RD}}=1$, no error floor occurs. For both systems the RAID scheme clearly outperforms the common scheme. But as the number of users increases, the overall performance is more and more dominated by the detection at the relays leading to a decreasing performance gap between both schemes.
As could be seen, frequency selectivity has a strong impact on the overall performance. While for given system parameters convergence of the detection process may be successful for flat channels, it might fail for frequency selective channels. This behavior could be observed in, e.g., Figure $9 \mathrm{a}$ and is mainly caused by the use of softRAKE detection. To overcome these drawbacks, the code rate of the repetition code can be decreased as it was done in Figure 9b,c. However, a lower code rate leads to a lower spectral efficiency which is not always desirable. Another possibility is the application of a different interference cancelation scheme like minimum mean square error (MMSE) based Interference Cancelation. In

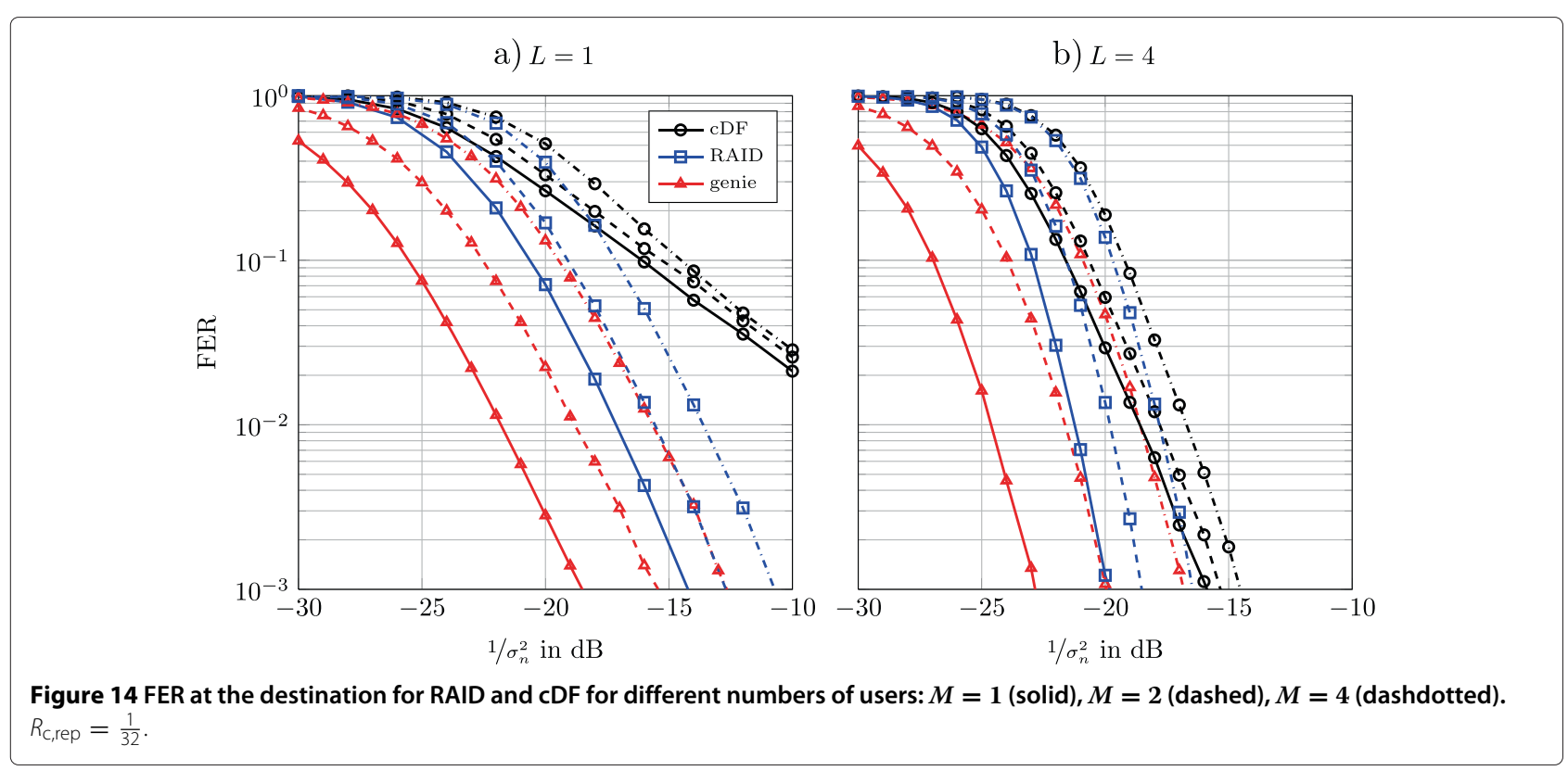


contrast to soft-RAKE detection, the MMSE detection resolves all multipath propagations of each signal jointly and, thus, does not suffer from frequency selectivity. However, since the MMSE based Interference Cancelation requires matrix inversions, its complexity is much higher than the complexity of the soft-RAKE detection [23]. Finally, a third possibility which avoids the drawbacks of both aforementioned methods is the combination of IDM-STCs and OFDM as it was investigated in [16]. The resulting OFDM-IDM-STCs allow for soft-RAKE detection in frequency domain independent of the number of channel taps. Since the detection of OFDM-IDM-STCs only requires a modification of the Interference Cancelation compared to the detection of IDM-STCs, the principles of the new proposed RAID scheme can directly be adopted for detection of OFDM-IDM-STCs.

\section{Conclusions}

In this article, distributed interleave-division multiplexing space-time codes (dIDM-STC) have been applied for multi-user two-hop relay systems. After introducing an equivalent transmission model for the source-relay transmission, the novel reliability-aware iterative detection scheme (RAID) was presented which explicitly takes the decoding success as well as the decoding reliabilities of the relays into account for detection at the destination. The proposed RAID scheme was shown to achieve substantial performance gains compared to the common detection scheme which neglects the reliability of the transmission on the first hop and implicitly assumes perfect decoding at the relays. Due to the optimal exploitation of all available information from the correct as well as from the erroneous relays, RAID was shown to even outperform the adaptive relay scheme in non-overloaded systems. Moreover, the impact of the estimation of the bit error probabilities at the relays using LLRs was investigated. It was shown that using these estimates for the RAID leads to almost the same end-to-end performance than the usage of perfect knowledge. Only for small frame sizes slightly higher frame error rates using the estimates could be observed, but for larger frames, practically no differences in terms of frame error rates could be seen. Finally, the combination of IDM-STCs and OFDM was suggested to better cope with frequency selectivity. For the resulting OFDM-IDM-STCs the principles of the RAID scheme can directly be applied in frequency domain.

\section{Competing interests \\ The authors declare that they have no competing interests.}

\section{Acknowledgements}

This study was supported in part by the German Research Foundation (DFG) under grant KA 841/20-2.
Received: 22 June 2012 Accepted: 16 November 2012

Published: 8 April 2013

\section{References}

1. G Foschini, M Gans, On limits of wireless communications in a fading environment when using multiple antennas. Wirel. Personal Commun. 6(3), 311-335 (1998)

2. E Telatar, Capacity of multi-antenna Gaussian channels. Eur. Trans. Telecommun. 10(6), 585-595 (1999)

3. M Dohler, Virtual Antenna Arrays, PhD thesis, University of London (2003)

4. S Alamouti, A simple transmit diversity technique for wireless communications. IEEE. J. Sel. Areas Commun. 16(8), 1451-1458 (1998)

5. V Tarokh, N Seshadri, A Calderbank, Space-time codes for high data rate wireless communication: performance criterion and code construction. IEEE Trans. Inf. Theory. 44(2), 744-765 (1998)

6. M Dohler, E Lefranc, H Aghvami, in IEEE International Symposium on Personal, Indoor and Mobile Radio Communications (PIMRC). Space-time block codes for virtual antenna arrays (Lisbon Portugal, 2002), pp. 414-417

7. J Laneman, G Wornell, Distributed space-time-coded protocols for exploiting cooperative diversity in wireless networks. IEEE Trans. Inf. Theory. 49(10), 2415-2425 (2003)

8. W Leung, K Wu, L Ping, in IEEE Vehicular Technology Conference (VTC-Spring). Interleave-division-multiplexing space-time codes (Jeju South Korea, 2003), pp. 1094-1098

9. K Wu, L Ping, A quasi-random approach to space-time codes. IEEE Trans. Inf. Theory. 54(3), 1073-1085 (2008)

10. L Ping, L Liu, K Wu, W Leung, Interleave-division multiple-access. IEEE Trans. Wirel. Commun. 5(4), 938-947 (2006)

11. Z Fang, L Li, Z Wang, in IEEE International Conference on Communications (ICC). An interleaver-based asynchronous cooperative diversity scheme for wireless relay networks (Beijing China, 2008), pp. 4988-4991

12. P Weitkemper, $P$ Wübben, KD Kammeyer, in IEEE International Symposium on Wireless Communication Systems 2009 (ISWCS). Distributed interleave-division multiplexing space-time codes for coded relay networks (Siena Italy, 2009), pp. 488-492

13. TWang, A Cano, G Giannakis, J Laneman, High-performance cooperative demodulation with decode-and-forward relays. IEEE Trans. Commun. 55(7), 1427-1438 (2007)

14. R Thobaben, in Asilomar Conference on Signals, Systems and Computers. On distributed codes with noisy relays (Pacific Grove CA, USA, 2008), pp. 1010-1014

15. H Sneessens, J Louveaux, L Vandendorpe, in IEEE International Conference on Acoustics, Speech and Sinal Processing (ICASSP). Turbo-coded decode-and-forward strategy resilient to relay errors (Las Vegas NV, USA, 2008), pp. 3213-3216

16. F Lenkeit, C Bockelmann, D Wübben, A Dekorsy, in 16th International ITG Workshop on Smart Antennas (WSA). OFDM-IDM space-time coding in two-hop relay-systems with error-prone relays (Dresden Germany, 2012), pp. 97-104

17. F Lenkeit, D Wübben, A Dekorsy, in IEEE 76th International Vehicular Technology Conference (VTC-Fall). An improved detection scheme for distributed IDM-STCs in relay-systems (Québec Canada, 2012), pp. 1-5

18. M Noemm, T Wo, P Hoeher, Mutilayer APP detection for IDM. Electron. Lett. 46, 96-97 (2010)

19. L Bahl, J Cocke, F Jelinek, J Raviv, Optimal decoding of linear codes for minimizing symbol error rate. IEEE Trans. Inf. Theory. 20(2), 284-287 (1974)

20. I Land, Reliability information in channel decoding. PhD thesis, Kiel, Germany (2005)

21. A Papoulis, S Pillai, Probability, Random Variables, and Stochastic Processes, 4th edn. (Mc Graw Hill, New York, 2002)

22. J Hagenauer, E Offer, L Papke, Iterative decoding of binary block and convolutional codes. IEEE Trans. Inf. Theory. 42(2), 429-445 (1996)

23. K Kusume, G Bauch, W Utschick, in IEEE Global Telecommunications Conference (Globecom). IDMA versus CDMA: detectors, performance and complexity (Honolulu, HI USA, 2009), pp. 1-8

doi:10.1186/1687-6180-2013-70

Cite this article as: Lenkeit et al:: Reliability-aware iterative detection scheme (RAID) for distributed IDM space-time codes in relay systems. EURASIP Journal on Advances in Signal Processing 2013 2013:70. 